\title{
Gastritis enfisematosa como manifestación de isquemia mesentérica no oclusiva tras coartectomía
}

\section{Emphysematous gastritis as a manifestation of non-occlusive mesenteric ischemia after coartectomy}

\author{
Deyanira Quiñonez-López ${ }^{1 *}$, Carolina Gutiérrez-Cortés², Catalina Lince-Rivera², César A. Zuluaga- \\ Orrego ${ }^{1}$ y Edgar Giovanny-Ríos ${ }^{3}$ \\ ${ }^{1}$ Unidad de Cuidado Intensivo Pediátrico; ${ }^{2}$ Servicio de Pediatría; ${ }^{3}$ Servicio de Cirugía Cardiovascular. Hospital Universitario San Ignacio, \\ Cundinamarca, Bogotá D.C., Colombia
}

\begin{abstract}
Resumen
Las intervenciones mayores en cirugía cardiovascular de pacientes con patología aórtica pueden traer como consecuencia el compromiso de la perfusión de órganos distantes al sitio de la cirugía, siendo el tracto gastrointestinal uno de los más sensibles a los cambios hemodinámicos, en especial en grupos extremos de edad con un alto riesgo de morbimortalidad. Se reporta el caso de una lactante con antecedente de síndrome de Turner, quien es llevada a corrección de coartación de aorta más hipoplasia del arco, presentando como complicación posoperatoria un cuadro de gastritis enfisematosa con compromiso sistémico, proceso que fue favorecido por bajo gasto cardiaco posterior a bomba y presencia de urosepsis temprana, todo lo cual fue interpretado como parte del espectro del síndrome de isquemia mesentérica no oclusiva. Su diagnóstico precoz hizo posible una pronta intervención, consistente en soporte nutricional parenteral, freno ácido, antibioticoterapia de amplio espectro y seguimiento radiológico estricto, logrando la resolución completa de su sintomatología, sin complicaciones a corto plazo. Este caso demuestra que la identificación de factores de riesgo de isquemia esplácnica, una alta sospecha clínica y un cuidadoso manejo médico permiten un desenlace favorable para una patología con una alta tasa de mortalidad y muy pocos casos reportados en población pediátrica.
\end{abstract}

Palabras clave: Coartación de aorta. Gastritis enfisematosa. Isquemia mesentérica. Neumatosis gástrica.

\begin{abstract}
Major interventions in cardiovascular surgery of patients with aortic pathology can result in the compromise of perfusion of organs distant from the surgery site, the gastrointestinal tract being one of the most sensitive to hemodynamic changes, especially in extreme age groups with a high risk of morbidity and mortality. The case of a young infant is reported, with a history of Turner syndrome, who is led to correction of aortic coarctation plus arch hypoplasia, presenting as a postoperative complication a picture of emphysematous gastritis with systemic compromise, a process that was favored by low cardiac output post-pump and the presence of early urosepsis, all of which was interpreted as part of the spectrum of non-occlusive mesenteric ischemia syndrome. Its early diagnosis made possible a prompt intervention consisting of parenteral nutritional support, acid brake, broad-spectrum antibiotic therapy and strict radiological follow-up, achieving complete resolution of her
\end{abstract}

\section{Correspondencia:}

*Deyanira Quiñonez-López

E-mail: adquinonez@ husi.org.co
Fecha de recepción: 26-04-2020

Fecha de aceptación: 16-05-2020

DOI: 10.24875/ACM.20000182
Disponible en internet: 03-11-2020 Arch Cardiol Mex. 2021;91(2):215-220 www.archivoscardiologia.com 1405-9940 / C 2020 Instituto Nacional de Cardiología Ignacio Chávez. Publicado por Permanyer. Este es un artículo open access bajo la licencia CC BY-NC-ND (http://creativecommons.org/licenses/by-nc-nd/4.0/). 
symptoms, without short-term complications. This case demonstrated that the identification of risk factors for splanchnic ischemia, a high clinical suspicion and careful medical management, allowed a favorable outcome for a disease with a high mortality rate and very few cases reported in the pediatric population.

Key words: Coarctation of the aorta. Emphysematous gastritis. Mesenteric ischemia. Gastric pneumatosis.

\section{Introducción}

La coartación de la aorta presenta un espectro de gravedad en el que la edad temprana en su presentación se correlaciona estrechamente con la gravedad de la obstrucción y los defectos asociados ${ }^{1}$. La complicación esplácnica con más frecuencia descrita posterior a la corrección quirúrgica de esta patología es el síndrome poscoartectomía, secundario a la presencia de arteritis necrotizante del lecho mesentérico, la cual está relacionada con la instauración de hipertensión arterial en las primeras 48 horas del posoperatorio. Sin embargo, se describe el espectro contrario, que corresponde al síndrome de isquemia mesentérica no oclusiva, el cual representa el $1-4 \%$ del total de las complicaciones posquirúrgicas cardiovasculares ${ }^{1-3}$. Ocurre típicamente en casos de hipotensión grave con extensión variable del compromiso intestinal ${ }^{3}$. Dentro de esta amplia gama, podría considerarse la gastritis enfisematosa, que aunque es una rara condición supone una de las manifestaciones más graves de los eventos isquémicos intestinales, con una mortalidad descrita de hasta el $61 \%{ }^{4}$.

Este trabajo presenta el caso de una paciente lactante menor con coartación de aorta grave, que es llevada a corrección quirúrgica total bajo circulación extracorpórea en el segundo mes de vida. Durante el posoperatorio cursa con síndrome de bajo gasto de etiología multifactorial, evidencia de sangrado gastrointestinal alto con repercusión hemodinámica y confirmación radiológica de gastritis enfisematosa. Presenta adecuada respuesta al manejo médico conservador, consistente en apoyo cardiorrespiratorio, soporte con hemoderivados, antibioticoterapia y soporte nutricional parenteral total. La paciente cursa hacia un lento proceso de recuperación, que permite su traslado a la unidad de alta dependencia y su posterior egreso sin complicaciones.

\section{Presentación del caso}

Paciente de sexo femenino, nacida a término, Ballard de 37 semanas, con diagnóstico antenatal de síndrome de Turner $(45 \mathrm{X})$ y coartación de aorta. Al momento del nacimiento se confirma por ecocardiograma la existencia de una cardiopatía congénita tipo coartación de aorta extensa, con compromiso del arco proximal y distal, diámetro de $2 \mathrm{~mm}$ y segmento coartado de $14 \mathrm{~mm}$, válvula aórtica bivalva, doble sistema de vena cava superior, conducto arterioso grande de $5.2 \mathrm{~mm}$ con shunt bidireccional y signos de hipertensión pulmonar moderada. La paciente es manejada desde el nacimiento con ayuno preventivo e infusión de prostaglandina. Una vez logra ganancia ponderal, a los 41 días de vida es llevada a cirugía para reconstrucción extensa del cayado aórtico con tejido nativo de la aorta distal y cierre del conducto, bajo circulación extracorpórea, con hipotermia profunda a $18{ }^{\circ} \mathrm{C}$ y arresto circulatorio con perfusión cerebral anterógrada protectora, con tiempo de bomba de 171 minutos, tiempo de pinzamiento de 43 minutos y tiempo de arresto de 43 minutos. En el posoperatorio inmediato se corrobora una infección urinaria por Escherichia coli productora de betalactamasas de espectro extendido, para lo cual se inicia el tratamiento con un carbapenémico. Cursa con estado de choque vasopléjico secundario a respuesta inflamatoria posterior a la bomba y cuadro infeccioso asociado, requiriendo soporte vasoactivo múltiple, esteroide sistémico ante la sospecha de insuficiencia suprarrenal del paciente crítico y apoyo diurético en infusión a altas dosis por instauración de oliguria. Se inicia a las 48 horas del procedimiento quirúrgico estímulo trófico con leche materna, con evidencia 24 horas después de emesis en cuncho de café y distensión abdominal. El primer control radiológico muestra mal patrón de distribución gaseosa, sin otras alteraciones. Permanece en ayuno por 3 días más y, previo a nuevo estímulo intestinal, se toma radiografía que evidencia neumatosis gástrica (Fig. 1). Por esta razón se mantiene el ayuno indefinidamente, se coloca una sonda siliconada para evitar perpetuar el daño de la mucosa gástrica y se inicia soporte con nutrición parenteral total y freno ácido con un inhibidor de la bomba de protones, completando en total cuatro episodios de sangrado gastrointestinal en cuncho de café a pesar del reposo intestinal. Sumado a esto presenta anemia secundaria con compromiso hemodinámico correspondiente al descenso de $3 \mathrm{~g}$ de la hemoglobina con respecto al ingreso, por lo que 


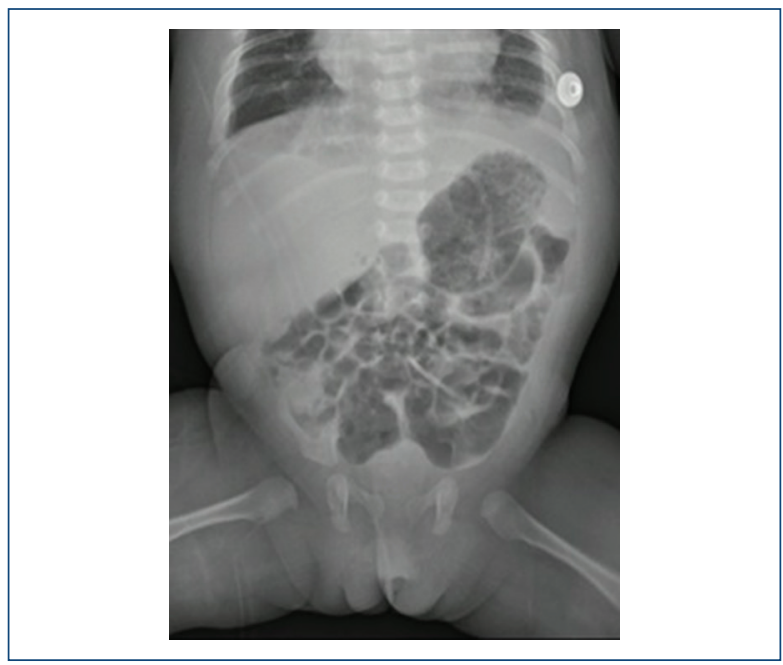

Figura 1. Neumatosis gástrica.

requiere transfusión de glóbulos rojos empaquetados. En total se realizan dos sets de hemocultivos, los cuales son negativos. La ecografía de abdomen no evidencia alteraciones. En manejo conjunto con cirugía y gastroenterología pediátrica, se decide la realización de control radiológico diario, con mejoría progresiva de la imagen (Figs. 2 y 3), y el reinicio de la nutrición enteral con fórmula extensamente hidrolizada hasta completar 7 días desde el último control radiológico normal. Se evidencia una adecuada tolerancia a la progresión de la vía enteral, sin nuevos eventos de distensión abdominal, vómito ni deterioro radiológico. Desde el punto de vista cardiorrespiratorio, permite el destete exitoso del soporte vasopresor y ventilatorio, requiriendo apoyo con betabloqueador dada la presencia de hipertrofia residual concéntrica del ventrículo izquierdo, con adecuada respuesta. Finalmente, es trasladada a la unidad de alta dependencia y luego egresa sin complicaciones.

\section{Discusión}

Se han implementado numerosos procedimientos quirúrgicos para reparar la coartación de aorta; entre ellos, la resección del segmento estrecho más anastomosis terminoterminal es una de las técnicas más empleadas en la población neonatal y en el grupo de lactantes menores. Desafortunadamente, muchos neonatos tienen algún grado de hipoplasia del arco aórtico, al igual que extenso tejido ductal en la aorta periductal, de tal manera que la simple resección y anastomosis no siempre es suficiente para asegurar

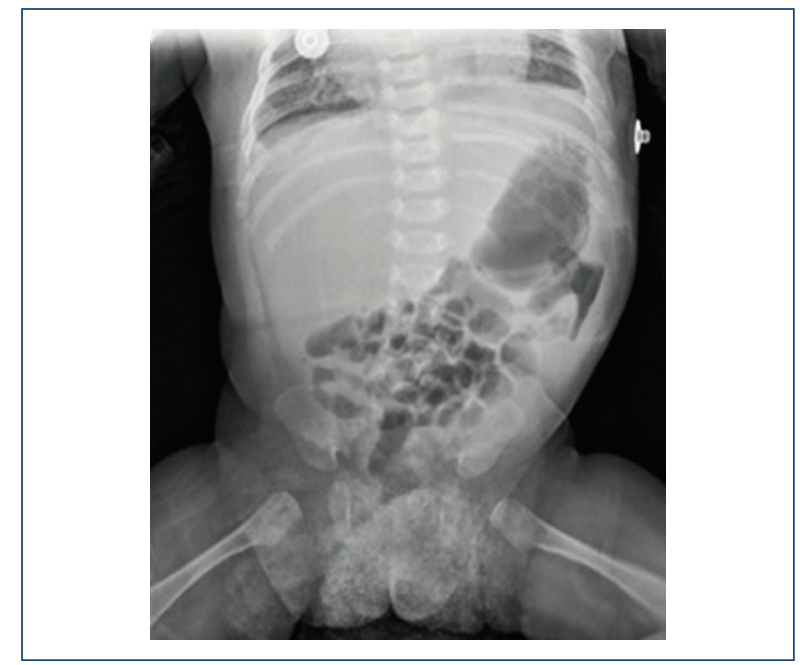

Figura 2. Neumatosis gástrica, tercio superior.

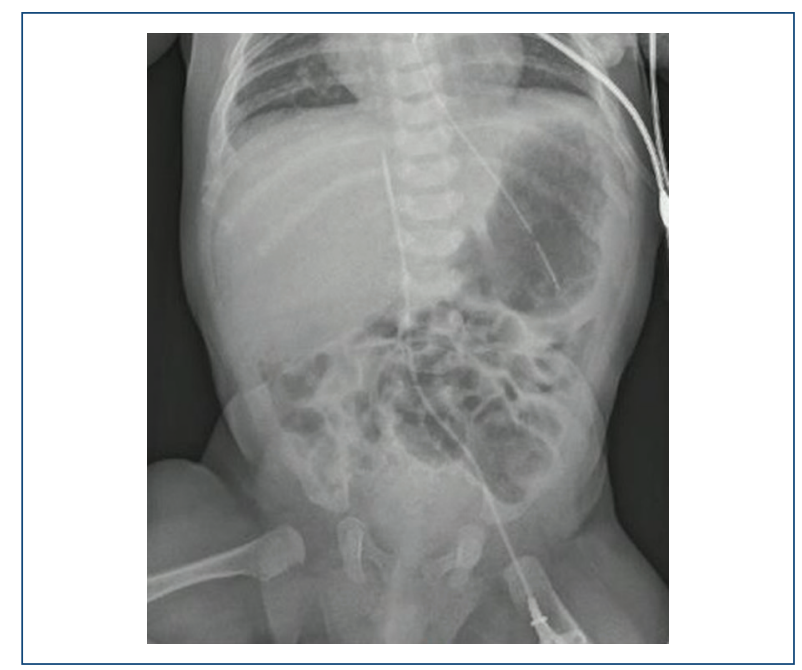

Figura 3. Neumatosis residual.

una adecuada liberación del sitio coartado ${ }^{1}$, como en el caso descrito, requiriendo intervenciones mayores bajo circulación extracorpórea. De igual forma, a pesar de la hipotermia profunda que teóricamente protege los órganos intrabdominales, este es un riesgo que se corre con todo el lecho esplácnico y en la actualidad no se cuenta con ninguna técnica en niños para proteger este lecho. Dado que los vasos femorales son de muy pequeño tamaño y no se pueden canular para dar protección y circulación retrógrada, tampoco es posible canular la aorta descendente y dar flujo distal, como se ha hecho en la población adulta. 
Dentro de las complicaciones quirúrgicas posteriores a este tipo de procedimientos cardiovasculares mayores en niños, la isquemia mesentérica no oclusiva (NOMI, Non-occlusive mesenteric ischaemia) representa el $1-4 \%$ del total, con una tasa de mortalidad del $60-100 \%{ }^{3}$. Fue descrita por primera vez por Ende, en 1958 , como el conjunto de todas las formas de isquemia mesentérica sin oclusión arterial. Aunque la fisiopatología exacta no está muy bien entendida, se asume que el mecanismo causal es una reducción extrema o una mala distribución del flujo esplácnico, con compromiso principalmente intestinal, aunque otros órganos también pueden ser afectados ${ }^{5}$. La isquemia da como resultado el compromiso de la integridad de la capa mucosa, con translocación bacteriana, bacteriemia y desarrollo de falla multiorgánica ${ }^{6}$. Dentro de los factores de riesgo para la instauración de NOMI se describen los siguientes: estado de bajo gasto cardiaco, falla renal con requerimiento de terapia dialítica, falla hepática, insuficiencia aórtica, uso de medicamentos vasoactivos que reducen el flujo esplácnico, cirugía cardiaca mayor o cirugía aórtica que requiera bypass cardiopulmonar, cirugía abdominal mayor y focos sépticos a distancia.

Con respecto a los mecanismos y mediadores de esta afección se han establecido:

- Falla en los procesos autorregulatorios necesarios para mantener la presión de perfusión intestinal en presencia de hipotensión sostenida.

- Vasoconstricción desproporcionada por el sistema renina-angiotensina-aldosterona y sus derivados, como mecanismo autorregulatorio en presencia de hipoperfusión intestinal.

- Elevación de la endotelina en respuesta a estados de choque, liberación de catecolaminas, angiotensina II y activación de la cascada de la coagulación.

- Producción incrementada de óxido nítrico, que actúa como radical libre e interfiere con la producción energética mitocondrial.

- Uso de alfa-adrenérgicos, especialmente norepinefrina a dosis $>0,1 \mu \mathrm{g} / \mathrm{kg}$ por minuto.

Se sabe además que en pacientes con suplencia sanguínea marginal en estado de choque y uso de vasopresores, en los que la extracción de oxígeno ha alcanzado su pico máximo, cualquier incremento en las demandas metabólicas sobre el enterocito para funciones tales como absorción, secreción y excreción aumenta el riesgo de isquemia ${ }^{5,7}$.

La NOMI leve ocurre sin síntomas manifiestos y se puede resolver sin secuelas, pero la NOMI grave se caracteriza por importante necrosis de la pared, perforación, peritonitis y muerte ${ }^{5}$. La presentación clínica típicamente incluye distensión abdominal, íleo, sangrado intestinal, fiebre, taquicardia y cambios en la coloración de la pared intestinal. Se requieren un alto grado de sospecha y valoraciones clínicas repetidas para hacer un diagnóstico temprano, el cual se podrá confirmar con radiografías de abdomen que muestran dilatación de asas, neumatosis intestinal, portograma aéreo y neumoperitoneo en caso de perforación ${ }^{3}$. Aunque se sabe que el íleon y el yeyuno distal son los más afectados ${ }^{6}$, la cavidad gástrica puede estar excepcionalmente comprometida y su manifestación más relevante es la presencia de neumatosis gástrica.

La neumatosis gástrica puede clasificarse en tres diferentes condiciones: enfisema gástrico benigno, gastritis flegmonosa y gastritis enfisematosa. En el enfisema gástrico benigno, el aumento de la presión intragástrica facilita la entrada de gas a través de áreas donde hay disrupción de la mucosa o alrededor de la vaina vascular y las áreas perilinfáticas, como en los casos de trauma de la pared gástrica o rotura de una bulla pulmonar. Radiológicamente el gas es de aspecto quístico, curvilíneo/lineal o burbujeante, los pacientes usualmente no lucen tóxicos, el tratamiento es conservador y el pronóstico es bueno una vez corregida la causa del aire intramural. Por otro lado, la gastritis flegmonosa consiste en inflamación de la pared gástrica debida a infección, pero sin aire intramural ${ }^{4}$.

Con respecto a la gastritis enfisematosa, se trata de una rara enfermedad descrita por Duo Vernoi en $1730^{2}$ y más tarde por Fraenkel en $1889^{4}$, en la cual se evidencia radiológicamente inflamación de la pared gástrica o se corrobora la presencia de gas intramural en el intraoperatorio, aunado a toxicidad sistémica, explicada esta por la formación de abscesos en la pared gástrica, en especial en la submucosa, con tendencia a drenar a la cavidad gástrica, y formación de celulitis gástrica que puede extenderse hacia la cavidad peritoneal con producción de peritonitis secundaria. En la radiografía de abdomen suele observarse un aspecto de burbujas en el interior de la pared del estómago (Fig. 1), similar a la neumatosis intestinal de la enterocolitis, con engrosamiento parietal y aumento de la densidad del tejido perigástrico debido a cambios inflamatorios e infecciosos. En algunas ocasiones se asocia a neumoperitoneo y neumatosis de la vena porta y sus ramas lobulares segmentarias intrahepáticas ${ }^{8-10}$. 
Desde el primer caso histórico reportado por Fraenkel se han registrado en total 68 casos, 59 de los cuales corresponden a pacientes mayores de 18 años ${ }^{4}$. Aunque más comúnmente se asocia con enterocolitis necrotizante y obstrucción intestinal distal, también se ha reportado en asociación con ingestión de ácido, gastritis erosiva, colocación intramural de una sonda de alimentación, fitobezoares, hematoma gástrico, eventos isquémicos y cirugía cardiaca mayor, como el caso aquí descrito $8,11,12$.

Esta afección se presenta generalmente en la primera semana después del evento desencadenante, aunque el tiempo de presentación puede prolongarse hasta 6 semanas después. La sintomatología incluye dolor abdominal epigástrico, náusea, vómito, diarrea, en algunos casos melena y hematemesis, con signos de inestabilidad hemodinámica y leucocitosis; síntomas que en su mayoría estuvieron presentes en nuestra paciente a las 48 horas del procedimiento y posterior al inicio del estímulo trófico con leche materna. El signo patognómonico de gastritis enfisematosa es la emesis de un coágulo de mucosa necrótica causado por disección bacteriana de la muscularis mucosae. Este fue un hallazgo característico en la menor que la llevó a descompensación hemodinámica secundaria al grado de anemización.

La fisiopatología exacta de la enfermedad aún no está bien dilucidada. Normalmente la mucosa gástrica protege las capas profundas del estómago mediante conexiones cerradas entre las células mucosas, la presencia de $\mathrm{pH}$ ácido y la suplencia de flujo sanguíneo, el cual conduce a una adecuada oxigenación y a la reparación tisular ${ }^{9}$. La disrupción de cualquiera de estas barreras puede explicar la instauración de la enfermedad. Las posibles etiologías para la lesión de la mucosa incluyen necrosis por licuefacción secundaria a ingestión de álcalis, necrosis por coagulación por ingestión de ácido, alteración en la barrera de la mucosa en pacientes con eventos isquémicos, cirugías gastrointestinales previas, abuso crónico de alcohol y focos sépticos a distancia, con posterior colonización e infección por diferentes microorganismos. Entre las bacterias más implicadas en la infección se describen E. coli, Streptococcus sp., Enterobacter sp., Pseudomonas aeruginosa, Clostridium welchi, estafilococos, Mucor sp. y Candida sp, entre otros ${ }^{12}$. No está claro de qué manera las bacterias productoras de gas entran a la pared gástrica, si es a través de una ulceración de la mucosa o por diseminación hematógena. No obstante, los procesos de reparación tisular que dependen de un adecuado riego sanguíneo se ven comprometidos en los casos de choque secundario a la liberación de citocinas proinflamatorias, posterior a procedimientos como el bypass cardiopulmonar, a cuadros infecciosos concomitantes y a una respuesta hipercontráctil de la vasculatura intestinal secundaria al uso de altas dosis de vasopresor, características que en su totalidad formaron parte del cuadro clínico de la paciente en mención, lo que seguramente facilitó el compromiso de la circulación esplácnica y la instauración de un proceso de NOMI con gastritis enfisematosa como manifestación clínica radiológica. Por último, cabe recordar que los procesos de isquemia intestinal pueden perpetuar la respuesta inflamatoria sistémica, lo cual conduce a un círculo vicioso que explicaría un desenlace fatal.

A pesar de que publicaciones previas han demostrado que los hallazgos radiológicos junto con una adecuada sospecha clínica son suficientes para hacer el diagnóstico, hasta el momento no hay un consenso general sobre la modalidad diagnóstica preferida. Sin embargo, la tomografía computarizada cuenta con la ventaja de su accesibilidad, buena sensibilidad y alta especificidad en la detección del gas, lo que la convierte en el método diagnóstico de elección ${ }^{12}$. En lo referente a la NOMI, a pesar de la posibilidad de detectar mala perfusión por tomografía, la angiografía se mantiene como el método de referencia para su diagnóstico temprano, siempre y cuando la condición médica del paciente permita su realización. Adicionalmente a la visualización directa del flujo mesentérico, el catéter insertado permite la infusión intraarterial mesentérica selectiva de fármacos vasodilatadores, tales como papaverina, nitroglicerina o glucagón en caso de isquemia crítica que requiera reperfusión del órgano ${ }^{6,13}$.

Si bien en los últimos 15 años ha habido avances en el manejo médico y endoscópico de esta enfermedad ${ }^{4}$, ningún estudio ha realizado un análisis sistemático para determinar los predictores de supervivencia e identificar las tendencias con respecto a la evaluación endoscópica de la gastritis enfisematosa. Dentro de las recomendaciones actuales de manejo de esta patología, se aconseja la monitorización del paciente en la unidad de cuidados intensivos, con miras a un tratamiento en lo posible conservador que incluya reanimación hídrica, soporte vasoactivo y ventilatorio en caso de choque, antibioticoterapia de amplio espectro, soporte transfusional y freno ácido con un inhibidor de la bomba de protones. La cirugía no está indicada a menos que haya evidencia de perforación o necrosis, o si el manejo conservador ha fallado, con una mortalidad reportada en algunas series superior al $60 \% 3,4,10$. 
El pronóstico es favorable si el diagnóstico ha sido precoz; conjuntamente a esto, se encuentra el inicio temprano de la antibioticoterapia acompañado de manejo de soporte ${ }^{14,15}$. Si la evolución es buena se produce la curación con hiperplasia del tejido conectivo y fibrosis residual o gastritis crónica, que se asemeja por su aspecto a la linitis plástica de Brinton ${ }^{10}$. De los pacientes que sobreviven, aproximadamente el 20$25 \%$ desarrollan estenosis gástrica que requiere dilatación ${ }^{4}$.

\section{Conclusiones}

La hipoperfusión esplácnica después de una cirugía cardiaca mayor, en especial del lecho aórtico, continúa siendo el enemigo silencioso, en especial en los neonatos y lactantes. En consecuencia, una evolución hemodinámica tórpida tras un resultado quirúrgico óptimo obliga a pensar en hipoperfusión de otros órganos, de los que el tracto gastrointestinal es uno de los de mayor riesgo. Pese a su rica irrigación, la cavidad gástrica puede comprometerse, expresándose como gastritis enfisematosa y, a su vez, como manifestación de NOMI. Es necesaria una alta sospecha clínica con miras a realizar una intervención médica temprana, que evite la posibilidad de complicaciones y la necesidad de intervención quirúrgica que claramente empeoran el pronóstico de los pacientes.

\section{Conflicto de intereses}

Los autores de este artículo manifiestan no tener ningún conflicto de intereses.

\section{Financiamiento}

La presente investigación no ha recibido ningún financiamiento específico de agencias de los sectores público, comercial o sin ánimo de lucro.

\section{Responsabilidades éticas}

Protección de personas y animales. Los autores declaran que para esta investigación no se han realizado experimentos en seres humanos ni en animales.

Confidencialidad de los datos. Los autores declaran que han seguido los protocolos de su centro de trabajo sobre la publicación de datos de pacientes.

Derecho a la privacidad y consentimiento informado. Los autores han obtenido el consentimiento informado de los pacientes y/o sujetos referidos en el artículo. Este documento obra en poder del autor de correspondencia.

\section{Bibliografía}

1. Hastings L, Nichols D. Coarctation of the aorta and interrupted aortic arch. En: Nichols D, Ungerleider R, Spevak P, Greeley W, Cameron D, Lappe D, et al., editores. Critical heart disease in infants and childrens. $2^{\text {nd }}$ ed. Philadelphia: Mosby Elsevier; 2006. p. 699-713.

2. Gil M, Balanzar J, Victoria G, Miguel M, López $H$, Ramírez A, et al. Diagnóstico de neumatosis gástrica en pacientes pediátricos. Reporte de series de casos. Acta Pediatr Mex. 2018;39:179-84.

3. Valencia M, Cassalett G. Sistema gastrointestinal y nutrición. En: Cassalett $\mathrm{G}$, editor. Manual de cuidado intensivo cardiovascular pediátrico. Bogotá: Distribuna; 2016. p. 155-67.

4. Watson A, Bul V, Staudacher J, Carroll R, Yazici C. The predictors of mortality and secuelar changes in management strategies in emphysematous gastritis. Clin Res Hepatol Gastroenterol. 2017:41:e1-7.

5. Al-Diery H, Phillips A, Evennett N, Pandanaboyana S, Gilham M, Windsor J. The pathogenesis of nonocclusive mesenteric ischemia: implications for research and clinical practice. J Intens Care Med. 2019;34:771-81.

6. Groesdonk H, Klingele M, Schlempp S, Bomberg H, Schmied W, Psych D, et al. Risk factors for nonocclusive mesenteric ischemia after elective cardiac surgery J Thorac Cardiovasc Surg. 2013;145:1603-10.

7. Sato $H$, Nakamura M, Uzuka T, Kondo M. Detection of patients at high risk for nonocclusive mesenteric ischemia after cardiovascular surgery. J Cardiothorac Surg. 2018;13:115.

8. Travadi J, Patole S, Simmer K. Gastric pneumatosis in neonates: revisited. J Paediatr Child Health. 2003;39:560-2.

9. Moosvi A, Saravolatz L, Wong D, Simms S. Emphysematous gastritis: case report and review. Rev Infect Dis. 1990;12:848-55.

10. Zamora M, Labarta L, Escos J, Cambra P, Vernal V, Seron C. Gastritis enfisematosa, eficacia del tratamiento con antibioterapia precoz. Gastroenterol Hepatol. 2016;39:393-402.

11. Murnan S, Miller J, Kuhn A. Gastric emphysema. A cannot-miss emergency medicine diagnosis. Pediatr Emerg Care. 2019;35:e152-3.

12. Cappa G, Silva R, Lisandrello N, Fadlala J. Gastritis enfisematosa como causa infrecuente de abdomen agudo. Rev Med Rosario. 2014; 80:129-34

13. Björck M, Wanhainen A. Nonocclusive mesenteric hypoperfusion syndromes: recognition and treatment. Semin Vasc Surg. 2010;23:54-64.

14. Lee S, Kim G, Kang D, Kim T, Song G, Kim S. Gastro-intestinal emphysematous gastritis. J Gastroenterol Hepatol. 2007;22:2036-9.

15. Yalamanchili M, Cady W. Emphysematous gastritis in a hemodialysis patient. South Med J. 2003;96:84-8. 